\title{
Legitimidad del uso del lenguaje filosófico en Teología
}

\author{
MARÍA JOSÉ CARAM \\ Universidad Católica de Córdoba (Argentina) \\ mariajosecaram@gmail.com
}

\begin{abstract}
Resumen
Siguiendo las reflexiones de M.-D. Chenu en Une école de théologie: Le Saulchoir, este artículo se pregunta por la legitimidad del uso del leguaje filosófico en teología. Para ello distingue el campo epistemológico propio de cada disciplina y establece el modo en que razón y fe se relacionan. Aunque es un hecho que en la historia de occidente la teología se expresó en un lenguaje filosófico, en el marco de la cultura contemporánea globalizada, se constata la emergencia de otras racionalidades en la articulación de los discursos sobre Dios.

Palabras clave: Filosofía, Teología, Encarnación, Crítica histórica, Interdisciplinariedad, Lenguajes.

\section{Legitimacy for the use of philosophical language in Theology}

Abstract

Following the reflections of M-D. Chenu in Une ecole de theologie: Le Saulchoir, this article poses the question of the legitimacy for the use of philosophical language in theology. For this he distinguishes the proper epistemological field of each discipline and establishes the way in which reason and faith are related. Although it is a fact that in the bistory of the West theology is expressed in a philosophical language, in the framework of globalized contemporary culture, one can confirm the emergence of other forms of reasoning in the articulation of discourse about God.
\end{abstract}

Key words: Philosophy, theology, Incarnation, Critical history, Interdisciplinarity, Languages.

Doctora en Teología por la Facultad de Teología San Vicente Ferrer, Valencia, España (2008). Profesora titular en el Departamento de Formación de la Universidad Católica de Córdoba, Argentina y Profesora Titular en el Centro de Estudios Filosóficos y Teológicos (CEFyT) Córdova, Argentina. Entre sus publicaciones se cuentan las siguientes: ¿Porqué las mujeres no? Espiritualidad de las peregrinas al Santuario de Qoyllur Rit'i, Cusco (1999); The Shape of Catholic Identity among the Aymara of Pilcuyo (1999); Manifestación del Espiritu en la fe de los pueblos andinos (2008); El Espiritu en el mundo andino. Una pneumatología desde los Andes (2012); Andar con Dios en la ciudad. Presencia del Espiritu en las prácticas de oración urbanas. Reflexiones a partir de un estudio de caso en la parroquia Santa María de la Paz (2014); Sumak Kawsay, Suma Qamaña o Buen Vivir. Una apuesta a la esperanza (2015); Tú eres Dios que me ve. Perspectivas para una reforma desde la diversidad de sujetos emergentes (2015); Un nuevo momento en la existencia de los pueblos indios. Crítica al indigenismo eclesiástico y nuevas perspectivas en el horizonte de la fe (2015).

Recibido: 24/Junio/2015 - Aceptado: 30/Julio/2015 


\section{Introducción}

La motivación para realizar este trabajo surge de un interés personal por profundizar, en el contexto de la conmemoración del $50^{\circ}$ aniversario del Concilio Vaticano II, en el legado de Fr. Marie Dominique Chenu (Soisy-sur-Seine, 1895-Paris, 1990), uno de sus teólogos más influyentes ${ }^{1}$. Particularmente, me interesa reflexionar sobre las razones que avalan el uso del lenguaje filosófico en teología según el pensamiento de este autor.

Para alcanzar este objetivo, circunscribiré mi reflexión en torno a un pequeño libro de Chenu titulado Une école de théologie: Le Saulchoir (1985), que, con toda razón, puede considerarse como "un ensayo teológico pionero" (Caram, 2014).

Le Saulchoir es, en efecto, una escuela de teología. Sin embargo la comunidad académica que lo conforma es consciente de estar viviendo un momento nuevo en el que la seriedad de los estudios exige estar a la altura de clima intelectual que se vive en aquella época y de las exigencias de un trabajo interdisciplinar serio. Por lo mismo, resalta en este opúsculo el valor de los métodos de trabajo y de la especificidad científica propios de las diferentes disciplinas que se desarrollaban en Le Saulchoir, a saber, la Filosofía, los Estudios Medievales y la Teología.

En lo que respecta a los estudios filosóficos y sus relaciones con la teología exigían en aquel momento una clara delimitación para superar los errores en los que había incurrido la escolástica moderna al deformar la especificidad y autonomía propias de la filosofía, convirtiéndola en un saber totalmente funcional a la teología (ancilla theologiae).

Une école de théologie: Le Saulchoir, fue publicado por primera vez en 1937 y, en 1985 fue reeditado por Les Éditions du Cerf, gracias a la iniciativa de G. Alberigo y del grupo Confrontations, quienes, pese al tiempo transcurrido, supieron valorar la actualidad de las reflexiones metodológicas allí contenidas. Entre las contribuciones que acompañan esta segunda edición del opúsculo se encuentra un artículo de Jean Ladrière (1985), titulado Théologie e bistoricité, al que me referiré explícitamente, pues contiene algunas reflexiones esclarecedoras para la problemática que intento abordar en estas páginas.

1 El presente escrito tiene su origen en un trabajo redactado recientemente con ocasión del curso de posgrado titulado "Problemas de legitimación en el lenguaje de la filosofía, de la religión y de la cultura contemporánea", dictado por el Dr. Gustavo Ortiz en la Facultad de Filosofía y Humanidades de la Universidad Católica de Córdoba durante el primer semestre del año 2013. 


\section{Un derrotero inesperado}

El opúsculo Une école de théologie: Le Saulchoir, tuvo un derrotero inesperado. Su origen se encuentra en las notas que el autor redactó con motivo de una conferencia que, como Rector, debía pronunciar ante la comunidad universitaria de Le Saulchoir el 7 de marzo de 1936, fiesta de Santo Tomás de Aquino ${ }^{2}$. La exposición fue muy bien acogida por profesores y estudiantes, a tal punto que muchos le rogaron publicarla. Respondiendo a esta petición, Chenu decidió redactar un texto más consistente, al que añadió algunas otras consideraciones sobre los temas metodológicos que iban definiendo el perfil de la escuela dominica francesa de teología. Aquella primera edición, a pesar de no haber tenido gran tiraje, llegó rápidamente a Roma y suscitó reacciones muy adversas. Pocos años más tarde, en 1942, Une école de théologie fue incluida en el índice de los libros prohibidos por un decreto del Santo Oficio y, su autor, destituido del cargo de Rector y despojado de su cátedra (Bosch, 2004: 257-258). ${ }^{3}$

El Concilio Vaticano II consagraría veinte años después las mismas orientaciones que habían costado la condenación del P. Chenu. La clarividencia de este teólogo se refleja en el siguiente párrafo en el que resuenan anticipadamente las intenciones de los Padres conciliares, expresadas especialmente en la Constitución Gaudium et Spes $\mathrm{n}^{\circ} 1$ :

Cuando, pues, queremos, con nuestro trabajo, estar presentes en nuestro tiempo, como lo estuvieron en el suyo un Tomás de Aquino o un Buenaventura, no es por un liberalismo invertebrado o por un oportunismo apologético. La misma ley del trabajo del espíritu y la comprensión que tenemos de nuestros contemporáneos, de sus problemas, de sus angustias, se convierten en criterio de auténtica objetividad (Chenu, 1985: 124) .

No es casual, por lo tanto, que Chenu haya sido uno de los teólogos rescatados por el Concilio. Aunque no participó en él como perito oficial, tuvo acceso a sus trabajos como asesor de un antiguo discípulo suyo, Mons. Claude Rolande, obispo de Madagascar. Y, desde esta

2 En esta fecha Le Saulchoir (nombre que significa lugar plantado con sauces) funcionaba todavía en Bélgica, en una antigua abadía benedictina, cerca de Tournai, en la localidad de Kain. Allí los dominicos franceses habían instalado en 1904, después de la expulsión de las Órdenes religiosas de Francia, su studium generale.

3 En otras publicaciones me detengo más extensamente sobre los puntos tratados en este párrafo. Ellas son: Caram (2012); Caram (2008) y Caram (2014).

$4 \quad$ Las citas de Une école de théologie: le Saulchoir han sido traducidas por la autora de este artículo.

VERITAS, No 34 (Marzo 2016) 
posición, así como también gracias a su estrecha vinculación con el P. Yves Congar, pudo influir en la redacción de algunos textos, particularmente en el primer mensaje del Concilio a la humanidad y en la teología de los signos de los tiempos (Franco, 2007: 67-68).

\section{La vida intelectual en Le Saulchoir}

La finalidad del opúsculo de Chenu es clara: reflexionar sobre los métodos de trabajo y las orientaciones espirituales que animan la vida y la labor intelectual del studium dominicano, que por aquel tiempo él dirige.

La tradición de la Orden de Predicadores y la inspiración de los padres Gardeil, Mandonnet y Lemonnyer, imprimieron a Le Saulchoir un sello característico: cultivar un trabajo intelectual con un espíritu de fidelidad innovadora, que se nutra tanto de la savia proveniente de las propias raíces medievales como de aquella que procede de los desafíos de la historia presente. Ni la situación de exilio vivida en Bélgica, ni la dispersión impuesta por la guerra de 1914, ni los conflictos propios de la crisis modernista lograron sofocar esta inspiración ni este impulso que, como decía el P. Gardeil, maestro por excelencia de esta escuela, los llevaba a pasar de un orden de los estudios fundamentales a "un orden de estudios conquistadores" (Chenu, 1985: 111) ${ }^{5}$.

En el primer capítulo de Une école de Théologie, Chenu ofrece una lectura retrospectiva de Le Saulchoir "como centro de reflexión y de investigación teológica e histórica, fiel a la inspiración tomista y comprometido con los problemas de la cultura contemporánea (Alberigo, 1985: 12). Se trata principalmente de una "escuela de teología", como lo indica el nombre de la obra que estamos considerando. Sin embargo sus trabajos no se circunscriben a esta disciplina. Precisamente, en función de ella y del compromiso con las problemáticas contemporáneas, la perspectiva que se asume es mucho más amplia y se relaciona con el clima intelectual del momento así como también con la organización de los estudios.

El rigor intelectual requiere un trabajo interdisciplinario en equipo, en el que juegan un papel muy importante los estudios filosóficos y medievales. Particularmente la responsabilidad de las dos Facultades, de Filosofía y Teología, así como también el Instituto de Estudios

5 La expresión, elocuente de por sí, indica una intuición inédita para aquella época y la consecuente decisión de llevar adelante un serio compromiso con un sustantivo desarrollo intelectual, el cual implicaba la renovación de los métodos de trabajo y un esfuerzo importante, que lograra otorgar a los estudios superiores su verdadero estatus en el marco de la reforma francesa de la enseñanza universitaria. 
medievales, les exige asumir con seriedad una reflexión sobre el estatus científico propio de cada disciplina para que, sin dar lugar a ninguna confusión, el saber tenga la posibilidad de avanzar haciendo frente a las nuevas problemáticas. En este sentido puede decirse que estamos ante una empresa pionera, pues, el trabajo interdisciplinar, aunque aún se encuentre en vías de maduración, ha adquirido carta de ciudadanía en nuestros días ${ }^{6}$.

Le Saulchoir se integra de lleno en el movimiento de gran desarrollo intelectual que toda Europa, Francia incluida, protagonizaba desde fines del siglo XIX. Esta tendencia reclamaba la renovación de los métodos de trabajo, con el fin de otorgar a la enseñanza superior su verdadera posición. Semejante aspiración exigía asumir el desafío de una investigación libre, de un trabajo desinteresado y la adopción de los instrumentos apropiados (Chenu, 1985: 111-112). León XIII supo animar y ratificar estas tendencias "tanto en el dominio filosófico como en los dominios bíblico, histórico y teológico" (Chenu, 1985: 112). La Revue des sciences philosophiques et théologiques, creada en 1907, refleja muy bien el compromiso con los debates contemporáneos de la Universidad laica así como también el rigor científico que supieron cultivar aquellos dominicos exiliados en Bélgica a principios del siglo XX, a pesar de encontrarse geográficamente lejos de la ebullición intelectual de la urbe ${ }^{7}$.

El 25 de marzo de 1923 la Sagrada Congregación de Estudios, concede a la Orden de Predicadores la autorización para entregar diplomas no sólo en teología sino también en filosofía. Con este hecho, queda reafirmada la autonomía alcanzada por los estudios filosóficos en las universidades eclesiásticas y se pone de manifiesto que no se los considera ya solamente como una propedéutica a la teología, sino como un ciclo completo con su propio equipamiento (Chenu, 1985: 112-113).

Por esta misma época, el P. Mandonnet introduce en el Saulchoir los estudios históricos, que luego darán lugar a la creación de un Instituto de Estudios Medievales. El interés por la historia se explica, en primer lugar,

6 Sobre la importancia de la interdisciplinariedad en teología puede consultarse el trabajo de Scannone (1990), titulado "Teología e interdisciplinariedad. Presencia del saber teológico en el ámbito de las Ciencias".

7 Cabe mencionar aquí que Santo Domingo envió a los frailes de la Orden recién establecida a estudiar y fundar conventos en los grandes centros urbanos del medioevo. Esta decisión implicaba indisociablemente la vida en comunidad y el trabajo en equipo, en colaboración con sus contemporáneos, en el corazón mismo de las nacientes universidades, donde se forjaba una nueva cultura. Chenu, en el primer capítulo de su opúsculo, da cuenta de esta intuición fundacional que, a lo largo de la historia de los Predicadores, fue capaz de fecundar los más valiosos intentos de renovación de la vida intelectual.

VERITAS, No 34 (Marzo 2016) 
por las mismas exigencias del trabajo teológico. Pero, además, porque precisamente en el Medioevo, se constituyó la teología católica latina y particularmente el pensamiento del Doctor Angélico. Por influencia de Mandonnet la crítica histórica introducida por el P. Lagrange en la Escuela Bíblica de Jerusalén comienza a aplicarse al estudio de santo Tomás, otorgando al Saulchoir un rasgo muy característico (Chenu, 1985: 113). Esta iniciativa es confirmada en 1921 por el P. Thessling, Maestro de la Orden, como respuesta al programa de especialización previsto por la ratio studiorum. El Instituto será canónicamente aprobado en 1937 por el decreto de la Sagrada Congregación de Estudios "que establece en Le Saulchoir las dos facultades de Teología y Filosofía según constitución apostólica de Pío XI "Deus scientiarum Dominum” en 1931 (Chenu, 1985: 113).

Por todo lo dicho, como afirma Alberigo, en la escuela del Saulchoir se sentía la necesidad de "hablar de métodos en plural, preferir el trabajo sobre las fuentes a la condenación de los manuales, tomar conciencia del valor relativo y no absoluto de los instrumentos conceptuales" (Alberigo, 1985: 17).

\section{Situación de la filosofía}

Reflexionar sobre la situación de la filosofía en un instituto que se autodefine como "escuela de teología" es un asunto delicado. Debe, por lo tanto, ser tratado cuidadosamente para no disminuir ni deformar la especificidad ni la autonomía que son propias de la ciencia filosófica, error en el que ha incurrido la escolástica moderna. Esta corriente de pensamiento, en efecto, se caracterizó por concebir a la filosofía y a la teología como una progresión científica y por usar de modo teológico a la filosofía (Chenu, 1985: 151-152), abusando del sentido medieval de la expresión ancilla teologiae.

Para una institución como Le Saulchoir, que tiene a su cargo dos facultades cuya finalidad es brindar una formación única y definitiva, la cuestión de la especificidad científica de cada una de ellas no puede dejarse de lado. Consciente de esta problemática, y haciendo uso de sus dotes de historiador, Chenu emprende la tarea de clarificar la especificidad científica y metodológica de la filosofía. Así, su preocupación principal en el capítulo 4 de Une école de théologie es dejar bien sentadas dos premisas.

La primera, es que se trata de dos universos diferentes. Así afirma Chenu que, "pasar de la teología a la filosofía, o viceversa equivale, en el plano del saber, pasar de un mundo a otro" (Chenu, 1985: 151). La segunda es que, en el contexto de un centro de estudios teológicos, la 
filosofía es parte de un todo, pero de ninguna manera deja de lado su especificidad. Si su finalidad es el desarrollo de una cultura (Chenu, 1985: 152), de ninguna manera se la puede reducir a la mera función propedéutica o instrumental. La razón filosófica, en efecto, cuenta con objeto, principios, métodos, e intereses propios que la habilitan para fundar un orden autónomo del saber. Y su autonomía científica es válida incluso dentro del pensamiento cristiano, pues confesarse tal no implica la renuncia a filosofar, como bien lo muestra la Suma Contra Gentiles de santo Tomás (Chenu, 1985: 153).

La confusión y la contaminación entre ambos órdenes del saber, se originan según Chenu, en los tiempos modernos y no en el medioevo, cuyos pensadores siempre han sido respetuosos de la distinción de los diferentes órdenes y procedimientos científicos. Es la escolástica moderna quien ha convertido a la filosofía en "una red de premisas y de conclusiones lógicamente dispuestas, construida anticipadamente, según una verdad, recibida totalmente hecha, con una fácil docilidad" (Chenu, 1985: 154), como si las soluciones existieran antes que los problemas y debieran aprenderse como una lección "sin drama y sin conquista" (Chenu, 1985: 154).

Los campos en los que se deja sentir esta nefasta influencia de la escolástica moderna son varios y merecen ser abordados aunque más no sea someramente para tomar conciencia de la magnitud de una problemática, que afectó tanto a las mutuas relaciones entre filosofía y teología como al vínculo del pensamiento católico con la cultura moderna y a la recta comprensión del legado de santo Tomás, el cual había quedado totalmente deformado con las 24 tesis tomistas que pretendían ser un resumen perfecto de las enseñanzas del Doctor Angélico, y se imponían a todos los candidatos al doctorado en teología (Bosch, 2004: 253).

\subsection{Filosofía tomista y escolástica barroca}

La distorsión de la filosofía tomista por la escolástica barroca se manifiesta en los siguientes aspectos.

\subsubsection{La adopción del concepto de philosophia perennis}

El vocablo philosophia perennis fue creado por Agustín Steuchus, filósofo del renacimiento, con el fin de reconciliar el teísmo paduano y la escolástica medieval (Ferrater Mora, 1971: 413). Si la expresión conserva su sentido para los teólogos, en cuanto que significa los preambula fidei o cimientos racionales de la fe, es inadmisible para calificar orgánicamente a la filosofía, pues compromete su actitud, su poder de sistematización y

VERITAS, No 34 (Marzo 2016) 
su originalidad investigativa. Chenu sugiere una verdadera caracterización de la philosophia perennis, correctiva de aquella perspectiva, que ha llevado a la pérdida de la percepción misteriosa de la verdad "manipulándola en pequeños paquetes bien hechos" (Chenu, 1985: 155). Y lo hace citando la siguiente frase de Platón: "Estamos en una situación crítica en la que es necesario examinar los objetos por todos lados, para sondear en ellos la verdad" (Chenu, 1985: 155).

\subsubsection{La concepción de lo inteligible}

La escolástica moderna concibe lo inteligible como un enlace de conceptos y todo el esfuerzo del pensamiento consiste en buscar su compatibilidad lógica. La verdad concebible, será comprendida como aquello en lo que es posible adquirir una idea clara y distinta, una noción. Así, la investigación y la ciencia consisten en alcanzar la definición del objeto mediante la demostración lógica. "El principio de razón suficiente, expresión de este ideal de análisis universal, es en sí mismo reductible a la exigencia de una identidad lógica y se remite al principio de contradicción” (Chenu, 1985: 155).

De este modo, desvinculada de los problemas de la existencia, de la acción, del devenir, del tiempo, queda encerrada en una filosofía de las esencias, donde sólo reina lo necesario, lo universal y las ideas inmóviles. La abstracción es la medida de su inteligibilidad, su método es exclusivamente deductivo y alcanza su perfección en "el "sistema", régimen de definiciones y de conclusiones donde lo irracional es objeto de sospecha, descartado como ininteligible" (Chenu, 1985: 155-156). Esta mentalidad caracterizaba a las mejores rationes studiorun y estaba presente en los manuales tomistas. Con ella, a beneficio de la perfección dialéctica, quedaba eliminado "el sentido del misterio, el gusto por la investigación y, hasta esta facultad de asombro que mantiene el espíritu abierto al progreso" (Chenu, 1985: 156).

\subsubsection{La ontología}

Como consecuencia de lo que acabo de afirmar, para los seguidores de la escolástica, la ontología se organiza según el "tercer grado de abstracción” y pierde su potencial contemplativo. La teodicea queda reducida a un sistema de pruebas físicas, sin trascendencia ni espiritualidad. Se desconfía tanto de los antiguos metafísicos de la participación como de las nuevas filosofías de la contingencia. Se niega el intelectualismo tomista contra la crítica de Bergson, se pierde la savia agustiniana medieval y la "mística dionisíaca". Su conceptualismo queda encerrado en un objeti- 
vismo positivista. Triunfa el formalismo lógico en detrimento de la curiosidad y se reemplaza la disputa medieval con los ejercicios escolásticos (Chenu, 1985: 156).

\subsection{Filosofía escolástica y filosofía moderna}

El antagonismo de la escolástica moderna se manifestó también frente a la filosofía moderna en los campos que menciono a continuación.

\subsubsection{El humanismo}

La primera manifestación del pensamiento moderno fue el humanismo, con el cual el tomismo pudo simpatizar, a pesar de que se encontraba en un estado de miseria extrema, según lo reconocían los mismos Capítulos Generales de la Orden dominicana (Chenu, 1985: 158).

Los Predicadores, en efecto, fueron sensibles a tres rasgos del nuevo movimiento filosófico: "el deseo de recobrar al Aristóteles auténtico, la admiración por Platón recientemente revelada, la preocupación por otorgar un valor religioso a la filosofía" (Chenu, 1985: 158). Aun contando con muchas excepciones, hay que decir que el convento dominicano de "Saint-Jacques tenía un buen entendimiento con las letras nuevas; y muchos dominicos de Florencia poseían un rango honorable en la historia de la lengua italiana" (Chenu, 1985: 158). "Los humanistas parisinos dice Chenu - abominaban la escolástica, estaban -ironía de las palabrasen contra de los moderni, es decir, de los nominalistas, sus contemporáneos, y por su alianza con los antiqui, tomistas o escotistas, triunfaron en París en los conflictos doctrinales de la Universidad (Chenu, 1985; 157).

Los escolásticos, en efecto, pretendiendo defender al humanismo medieval mediante la defensa de cierta cultura clásica dejaron de lado la creatividad y a la apertura propias del medioevo (Chenu, 1985: 156). De este modo, asumiendo una actitud totalmente contraria a la de santo Tomás, quien supo aprovechar el aporte del movimiento filosófico del siglo XIII, perdieron la oportunidad de enriquecerse con la orientación espiritual del Renacimiento (Chenu, 1985: 158).

\subsubsection{La ciencia moderna}

Otro rasgo del mundo moderno está dado por el nacimiento de un nuevo tipo de inteligibilidad a partir del desarrollo de las ciencias experimentales y por el papel que en él jugaron las matemáticas (Leonardo, Copérnico, Galileo, Descartes, entre otros). Fenómeno ante el cual, la 
metafísica tradicional dio muestras de falta de creatividad y de decadencia por su negativa a reconocer el mundo nuevo que se abría ante sus ojos (Chenu, 1985: 159). En este fallo mortal estuvieron involucrados muchos discípulos de santo Tomás, quienes, por observancia estricta de la letra y por infidelidad a sus propios principios metodológicos, practicaron con torpeza el concordismo. Llegaron a confundir las especies biológicas con las aristotélicas, pretendieron refutar con argumentos hilemórficos las teorías químicas y condenaron el relativismo de Einsten en nombre de los primeros principios (Chenu, 1985: 159).

\subsubsection{La crítica kantiana}

La crítica kantiana fue una amenaza de ruptura mayor, por el hecho de haber abierto camino al idealismo implícito aún en Descartes. En el conflicto desatado entre el idealismo y el realismo, los medievales jugaron un buen papel, ubicándose en el estado mismo de la cuestión, lo cual les permitió realizar una profundización audaz de sus principios. Así pudieron, "mediante una revisión de las fuentes profundas de su filosofía, superar las aporías del idealismo, y, si se puede decir, sacar provecho" (Chenu, 1985: 160). De este modo, los maestros tomistas del siglo XX,

a pesar de algunas divergencias de detalle y de expresión, pensaron que su filosofía "salvando el valor del conocimiento de las cosas por un método verdaderamente crítico, permite explorar en la intimidad del universo de la reflexión, y establecer allí, si así se puede decir, la topología metafísica; de este modo 'la filosofía del ser' es al mismo tiempo, por excelencia, 'una filosofía del espíritu'(J. Maritain) (Chenu, 1985: 160).

Chenu cita, entre los mejores trabajos producidos sobre el tema el Essai d'une étude critique de la connaissance, de Gosselin, (1932) autor que ejerció una gran influencia en el Saulchoir, al considerar que,

conforme al tomismo más auténtico que pueda admitirse, que todo juicio, el más elemental y el más evidente, supone un mínimo de reflexión, y de reflexión crítica, de la inteligencia sobre su acto, y por su acto, sobre su naturaleza. Debe, por lo tanto, continuarse a plena luz esta reflexión elemental, hacerla explícita y distinta, y esperar de ella un conocimiento más claro y riguroso del derecho que tiene la inteligencia para afirmar lo real (Chenu, 1985: 160).

Quedan, no obstante, en este posicionamiento frente al idealismo, algunas lagunas o límites. En orden a su superación, corresponderá a los historiadores una adecuada valoración de las descuidadas fuentes origina- 
les, en orden a hacer justicia al renacimiento agustiniano y a realizar "una purificación del racionalismo wolfiano, justamente atacado por la Crítica" (Chenu, 1985: 160).

Finalmente, diremos, siguiendo a Chenu, que la irrupción de la Crítica en el horizonte de la modernidad merece una valoración positiva. Ha sido, en efecto, una buena oportunidad para un renacimiento espiritual, a partir del "consentimiento al ser" y de la recuperación de una "sabiduría" fundada en el conocimiento de nuestro propio espíritu y de su libertad (Chenu, 1985: 161).

\subsubsection{La historia}

El siglo XIX aportó al mundo del conocimiento una apertura a la realidad del tiempo, la cual, acogida por muchas iniciativas filosóficas, lleva en sí la promesa de evitar nuevas rupturas con los "modernos". El tomismo posee muchas posibilidades de realizar aportes en orden a superar la triple barrera de "una escolástica estropeada por su propia complacencia, la rutina pedagógica y el uso de la autoridad (Maritain)" (Chenu, 1985: 161).

La barrera de las incompatibilidades seculares caerá, dice Chenu, "en la medida en que el tomismo esté convencido de la heterogeneidad de los tipos de saber, y por lo tanto, de la infinita flexibilidad del espíritu" (Chenu, 1985: 161). En este sentido resulta esclarecedora una sentencia de Alberto Magno que, embriagado por la ciencia griega recién descubierta exclamó: Non omnes scientiae demonstrativae adbuc inventae sunt ${ }^{8}$ (Chenu, 1985: 161).

\section{Teología y lenguaje filosófico}

Si filosofía y teología constituyen, como se dijo arriba, dos mundos diferentes y, por lo tanto, dos regímenes de inteligibilidad (Chenu, 1985: 151), cabe preguntarse, como lo hace Jean Ladrière ¿por qué la teología hace uso, en algunas ocasiones, del lenguaje filosófico y especulativo? ¿Por qué la tradición cristiana no permaneció en el uso del lenguaje bíblico? ¿Por qué la teología no elabora una construcción conceptual totalmente nueva? (Chenu, 1985: 65-66). Estas preguntas, formuladas sobre el telón de fondo de las difíciles cuestiones suscitadas en el conflicto en-

\footnotetext{
8 "No todas las ciencias demostrativas están constituidas todavía" (Traducción del latín ofrecida gentilmente a la autora por la Dra. Flavia Dezzutto).
}

VERITAS, No 34 (Marzo 2016) 
tre la cultura moderna y la escolástica barroca arriba mencionadas ${ }^{9}$, requieren, por una parte, esclarecer cuál es la situación propia de la teología $y$, por otra, ubicar correctamente el papel que juega el lenguaje en la construcción del pensamiento teológico.

En el fragor de la lucha anti-luterana, la teología perdió su conexión natural con su fuente viva que es la fe. De este modo, comienza a considerarse como "suficiente el asentimiento puramente formal a la enunciación dogmática. De este modo, el no creyente puede construir sobre ella un buen argumento, si su silogismo respeta la ley de los tres términos" (Chenu, 1985: 131). Razón suficientemente grave como para emprender "una obra de saneamiento de la teología", como sostenía el P. Gardeil, antecesor de Chenu en el gobierno del Saulchoir (Chenu, 1985: 130). En beneficio de esta tarea resultaba muy esclarecedora la distinción de los objetos formales, propia de la pedagogía tomista. Recurrir a tal distinción ayudaría, en efecto, a superar la confusión de ideas, métodos y vocabularios en que incurrieron los teólogos en el transcurso de la crisis modernista (Chenu, 1985: 139).

La teología nace de la fe, su origen debe buscarse siempre en el fides auditus y su objeto en la propia automanifestación de Dios. Se impone, por lo tanto, en primer lugar la afirmación de la primacía del dato revelado, es decir, de la Palabra de Dios, que ha de ser no sólo la regla, sino también el alma de la especulación teológica.

De ahí que la razón teológica sólo pueda desempeñarse allí donde está la luz de la fe, único medio capaz de garantizar "la indispensable homogeneidad espiritual... que va desde la comunión con el pensamiento divino hasta la última conclusión teológica" (Chenu, 1985: 130). En esta concepción, "la construcción especulativa no será ya un edificio conceptual superpuesto a una percepción previa y adquirida de una vez por todas. Es incorporación viva de la luz de la fe en las estructuras racionales" (Chenu, 1985: 131).

Las razones y deducciones no tienen la finalidad de enriquecer lo revelado y tampoco gozan de la facultad de hacerlo. El "dato" es algo recibido gratuitamente, y "la sistematización teológica más perfecta no agrega un ápice de luz y ni de verdad al Evangelio” (Chenu, 1985: 131).

El dato revelado es entregado al creyente por el camino de la historia. En la sucesión de las edades y en toda la vida positiva de la Iglesia (no en la metafísica) radica el realismo cristiano. Por lo mismo, y consi-

9 La ubicación histórica de la cuestión queda claramente formulada en el opúsculo de 1937, donde Chenu, posiblemente citando a Gardeil, dice: "la posición de las disciplinas teológicas en la que nos encontramos es la de los siglos XVI-XVII, y no en la de las Sumas medievales” (Chenu, 1985: 129). 
derando que en este proceso revelador de la Palabra de Dios, la Escritura y la Tradición ocupan un lugar preeminente, el estudio de las fuentes adquiere en Le Saulchoir el rango de una disciplina mayor. Corresponde, en efecto, al historiador proveer al teólogo, no de argumentos probatorios, sino de los textos testimoniales de la vida de la Iglesia, en donde también late verdadera la Palabra de Dios. En este sentido resultan esclarecedoras las palabras de Chenu cuando dice, remitiéndose a santo Tomás, que "los textos son a la teología lo que la experiencia es a la ciencia", es decir "la tierra sobre la que sin cesar renueva la vida y progresa en inteligibilidad". (Chenu, 1985: 132).

Según lo dicho, si la teología se aleja de la fe, pierde consistencia, pero de la fe arraigada en la experiencia creyente surge y crece "un apetito exigente, el intellectus fidei, que con una curiosidad permanente por el dato revelado, provoca una estima religiosa y científica de las disciplinas que lo elaboran" (Chenu, 1985: 134).

La consideración del vínculo entre teología e historia, de la historicidad propia de la fe que busca entender, dará pie para afrontar el problema del lenguaje teológico.

Se ha dicho que la teología sólo puede nacer de la fe. Ahora bien, por esta misma fe, el creyente ofrece su asentimiento a proposiciones que de suyo son solidarias con la historia y que son recibidas, no producidas de ninguna manera por un trabajo científico. Estas proposiciones y enunciados no son sino la propia realidad divina expresada en lenguaje humano. La ley de la encarnación se manifiesta aquí (Chenu, 1985: 139).

La teología no se expresa necesariamente en un lenguaje filosófico. Sin embargo, dice Ladrière, la historia de occidente "nos enseña que, de hecho, sobre la base del lenguaje originario, del lenguaje de la Biblia, se han desarrollado diversas formulaciones que sólo fueron posibles gracias a un trabajo teológico" (Ladrière, 1985: 65). La Iglesia, en efecto, para resolver los cuestionamientos y dudas, para hacer frente a las controversias, ha debido utilizar los mismos términos en los cuales los problemas fueron planteados, de tal modo que el trabajo teológico desplegado quedó incorporado a la misma formulación de la fe (Ladrière, 1985: 65).

Si esta reflexión sobre la naturaleza, métodos y lenguaje de la teología responde a la necesidad de validar su condición después de la decadente etapa barroca, la tarea de legitimación del trabajo teológico de ninguna manera implica menoscabar los esfuerzos que la razón despliega para acercarse a la comprensión de un misterio siempre inalcanzable. Chenu dirá que este trabajo es exigido por la fe porque "la fe reside en la razón” (Chenu, 1985: 144). En ella vive y por ella actúa y su presencia en la potencia racional se explica por la ley de la encarnación de la Palabra de Dios. Presencia misteriosa de la fe en la fragilidad, no sólo de la razón 
humana, sino también en todo lo que ella produce. Con toda lucidez la idea rectora del método histórico-crítico de la escuela del P. Lagrange, sostiene que esta encarnación "se realiza incluso en la letra de los vocablos -'inspiración literal', se dice-, y la propia letra con toda su factura gramatical, literaria, histórica, es en realidad la vía de acceso a la inteligencia de la fe. La Palabra de Dios está en la palabra humana" (Chenu, 1985: 241).

Si el acto del creyente no termina en una proposición dogmática sino en el misterio que la envuelve y le da consistencia, es necesario que la razón ponga todos sus recursos al servicio del fides quaeres intellectus, según la propia economía de Palabra revelada, la cual "habla humanamente, reviste las formas del espíritu humano y se manifiesta en él según todas las condiciones exigidas por el mecanismo de una psicología ligada a la lenta y pesada complejidad de nociones y juicios, ligada a las categorías de tiempo y espacio" (Chenu, 1985: 136).

La fe así encarnada, busca su propia comprensión y, para ello, pone en marcha todo el dinamismo natural de la razón, y aplica necesariamente todos los medios que ella le suministra para remitirnos a una experiencia sui generis sobre la que trabaja la filosofía, y que constituye el marco de la elaboración conceptual: descubrimiento de la capacidad instauradora del lenguaje como medio productor de sentido, posibilidad de argumentación y demostración propiamente dicha. A continuación, conciencia de la posibilidad que se tiene de llegar hasta los principios, para dar razón de los fenómenos, explicándolos y uniéndolos mediante vínculos deductivos. Luego, otorgando la posibilidad de someter estas operaciones a reglas con sus propios principios y, también, de juzgar la validez de estas reglas. Finalmente, dando lugar al descubrimiento de la idea de fundamento.

Todo esto es expresado por la razón mediante un lenguaje, vehículo de lo inteligible, cuya estructura lógica proporciona al concepto posibilidades finitas. "Lo que la fe moviliza cuando utiliza los recursos que le brinda el concepto es, a través de éste, ese orden creado, con todas sus potencialidades, en sus posibilidades pero también en su finitud." (Ladrière, 1985: 67). El orden conceptual no es infinito. Por lo tanto, impone de algún modo a la fe sus propias limitaciones.

Como lo dice el mismo Chenu, "el creyente sabe que su fe no trabaja sino en y desde opciones racionales; los instrumentos especulativos han devenido factores decisivos de esta inteligibilidad, de modo que sus conclusiones no son sino el humano y frágil contexto del misterio, reflejo de la fe en un pensamiento autónomo" (Chenu, 1985: 147).

La razón, a través de todo su dinamismo y de todas sus operaciones, tiene una tarea propia: proporcionar a la existencia la comprensión de su 
propio ser (Ladrière, 1985: 69). La fe, por su parte, se inserta en la existencia, y desde este lugar, busca aclarar su propio objeto, la Palabra de Dios, sirviéndose de los conceptos que están ligados a la interpretación que la existencia se da a sí misma. Para que este quehacer sea posible, es decir, para que los conceptos puedan ser retomados efectivamente en el lenguaje de la fe, dice Ladrière, deben darse dos condiciones.

La primera es que debe haber cierta correspondencia entre los órdenes, más precisamente, una relación de analogía entre el orden existencial y el orden de la gracia que en él se revela y manifiesta. Esta analogía se funda sobre una relación real entre los dos órdenes, pues Dios que se revela no es totalmente extraño a la existencia y, además, responde a la pregunta que ésta lleva en sí, a esa espera de su cumplimiento que constituye su estructura esencial. "Lo que se cumple en el orden de la gracia es la realización misma del deseo más secreto de la existencia" (Ladrière, 1985: 70).

La segunda, es lo que se denomina "excedencia del concepto", es decir, una capacidad propia que le permita desbordar su campo original de significación. Estas dos condiciones, por otra parte, están ligadas siempre que la excedencia del concepto concierna a la analogía, la justifique, y funde la posibilidad de realizar transposiciones conceptuales. De hecho, un concepto no opera sino relativamente en un horizonte y no tiene una significación particular sino en relación con otros conceptos. En este reenvío conceptual mutuo, en esta articulación lógica se constituye un horizonte propio, que define la filosofía y que "es planteado por el despliegue mismo del proyecto filosófico" (Ladrière, 1985: 71).

La teología, sin embargo, abre otro horizonte o, como se ha dicho anteriormente, otro mundo. Se produce, entonces, en virtud de la analogía de los órdenes, una especie de superación de significaciones: "la significación que recibía el concepto en el horizonte filosófico, en el cual fue constituido, es transmutado a otra significación que, a la vez retoma, prolonga y transforma la primera, en el horizonte teológico en el cual se inserta" (Ladrière, 1985: 71). De este modo, la excedencia del concepto se funda en la articulación de horizontes. Pero no es más que un aspecto específico: la comprensión y la articulación de órdenes.

La razón, en efecto, no se agota en el trabajo filosófico. Hay otras figuras de la razón, como por ejemplo, la de la razón científica en particular. Ella presupone un espacio de representación que culminando en el modelo matemático rompe la reflexión y la adhesión de la existencia a sí misma que la reflexión implica. Ahora bien, si la fe dice relación con la existencia, "la forma de razón sobre la cual la fe puede apoyarse es la de la auto-reflexión de la existencia" (Ladrière, 1985: 71). 
La interacción entre filosofía y teología, y entre filosofía y formulación dogmática, se esclarece mediante la consideración de algunas cuestiones actuales. Tanto la filosofía como la teología poseen una historicidad propia. En esta historicidad hay que distinguir un componente constitutivo (interno) y un componente de interacción (externo). En el caso de la teología el componente interno es la vida de la Iglesia y consiste, como lo ha señalado Chenu, en "estar presente en el propio tiempo", lo cual teológicamente hablando significa "estar presente ante el dato revelado en la vida actual de la Iglesia y en la experiencia actual de la cristiandad" (Chenu, 1985: 142).

Entre los temas inventariados por Chenu, que conservan actualidad en 1985, año en que escribe Ladrière la reflexión que estoy siguiendo, pueden mencionarse particularmente tres: el pluralismo de civilizaciones, el movimiento social y la participación de los laicos en el apostolado jerárquico. A ellos se agregan otras cuestiones emergentes, llamadas a ser integradas en la reflexión teológica, y que de hecho actualmente lo están siendo: los problemas éticos ligados a la ciencia y a la vida política, la erosión de los basamentos naturales y sociales de la religión y, finalmente, la problemática del cosmos, dominio en el que la obra de Teilhard de Chardin ha abierto un camino muy interesante.

Todas estas problemáticas están siendo expresadas en las diferentes búsquedas de Dios que tienen lugar en nuestro tiempo. De hecho, pareciera que la teología posconciliar ha inaugurado una época de oro, según la teóloga norteamericana E. Johnson (2008: 17). El panorama de la teología contemporánea se ha enriquecido notablemente con la interacción entre las culturas. Fenómeno cada día más evidente en la era de la globalización, que conlleva el desafío de pensar la pluralidad, no sólo en el ámbito de la cristiandad latina, sino a nivel mundial. Surgen nuevos lenguajes sobre Dios, enraizados en otras tradiciones culturales y en otras formas de pensamiento que muchas veces no utilizan un lenguaje filosófico para expresarse.

Un panorama bastante amplio de esta situación es presentado por E. Johnson en su obra La búsqueda del Dios vivo. Trazar las fronteras de la teología de Dios (2008). Representantes significativos de estos esfuerzos son los teólogos de la liberación, entre los que sobresale G. Gutiérrez. La teología india que crece en América Latina cuenta con los trabajos de E. López Hernández $(1993 ; 2000 ; 2004)$ y con un proceso de reflexión que se realiza colectivamente a través de encuentros ecuménicos regionales y continentales que se llevan a cabo periódicamente. La teología feminista, por su parte, ha realizado enormes y valiosos avances, expresa la necesi- 
dad de hablar de Dios desde otro modo de sentirlo (De Prado, 1994) ${ }^{10}$. Cabe mencionar también las teologías elaboradas en contextos africanos y asiáticos ${ }^{11}$. Estos discursos, articulados desde racionalidades muy diferentes a la que Occidente ha valorado, emergen y luchan por validarse en un contexto eclesial muchas veces adverso y se van construyendo en una dinámica de diálogo intercultural, ecuménico e interreligioso.

El movimiento social, por su parte, se ha ampliado considerablemente con una cada vez mayor toma de conciencia, no sólo de los pobres de los países del llamado Tercer Mundo, sino con la voz de los excluidos y de las minorías, que van enriqueciendo los discursos teológicos a partir de sus propias experiencias de sufrimiento y de sus propias especificidades étnicas, raciales, religiosas, de género, etc. En los orígenes de la Teología de la liberación, la experiencia compartida en el esfuerzo por la abolición de la injusticia y por la construcción de una sociedad más libre y más humana, jugó un papel importantísimo (Gutiérrez, 1996: 6162), aportando una manera original de pensar la encarnación y las relaciones entre fe e historia.

Finalmente, la participación de los laicos en el apostolado jerárquico ha tenido un derrotero bastante complejo bajo los últimos pontificados, y en nuestros días resurge con nuevos planteamientos. En este campo resalta el tema pendiente de la participación de las mujeres en la vida de la Iglesia.

\section{Conclusiones}

Une école de Théologie puede considerarse, por todo lo dicho, una obra pionera pues muchas de sus intuiciones, maduradas en el contexto de un centro de estudios específicamente teológico y dominicano, abierto a los debates de la cultura de su época, han seguido un hilo de desarrollo que perdura y se enriquece en nuestros días.

La gran crisis modernista puso en cuestión la validez de los métodos y lenguajes utilizados en las escuelas católicas de teología de la época, infectadas por los vicios intelectuales de una escolástica decadente. Ante esto, el equipo de Le Saulchoir pudo situarse en la posición misma del

10 Una muestra del importante desarrollo alcanzado por el quehacer teológico de las mujeres se encuentra publicado en la colección en tres volúmenes titulada Mujeres haciendo teologías, coordinada por la Dra. Virginia Azcuy y otras colaboradoras, en el que se recoge la labor teológica de teólogas de América Latina, el Caribe y Estados Unidos.

11 Como ejemplos de este teologizar desde el contexto asiático menciono particularmente la Teología del Pluralismo Religioso de Dupuis (2000) y las obras de Amaladoss (2000) y Pieris (2001).

VERITAS, No 34 (Marzo 2016) 
problema que se planteaba, gracias a una organización del trabajo intelectual arraigada en la tradición de una Orden medieval y, al mismo tiempo en el suelo de los movimientos culturales contemporáneos. El medioevo, y particularmente santo Tomás, le suministró las herramientas para superar las dificultades planteadas por las relaciones entre fe y razón. La inserción en los movimientos culturales y sociales, incluso en la misma vida cotidiana, le dieron la frescura de la fe vivida.

Las reflexiones sobre los métodos y objetos de trabajo, la distinción de los campos propios de cada disciplina, la asunción del método histórico-crítico aplicadas a los estudios tomistas permitieron a la escuela de Le Saulchoir validar su función tanto en el mundo de la cultura como en el de la vida de la Iglesia.

En lo que se refiere particularmente al lenguaje propio de la teología, la distinción epistemológica con la filosofía, la adopción de la crítica histórica en el trabajo teológico, la importancia otorgada a las fuentes, la inserción en las problemáticas sociales y, sobre todo la atención prestada a los instrumentos conceptuales fueron, entre otros factores, decisivos para liberar al discurso sobre Dios del enclaustramiento opresivo al que lo había sometido el racionalismo barroco y la burda simplificación del pensamiento de santo Tomás.

Nos preguntábamos al principio de este trabajo por las razones del uso del lenguaje filosófico en teología. Al concluir estas reflexiones, puede decirse que se trata de una situación no universalizable, porque de hecho ha tenido lugar en los ámbitos de influencia de Occidente. La Iglesia, en el ejercicio de su misión de anunciar el Evangelio, debió enfrentar muchas dificultades y se vio en la necesidad de dar razones de su fe. Por eso la formulación dogmática, portadora ella misma de revelación, asume los términos de los problemas que se fueron planteando en el curso de los tiempos.

El frondoso desarrollo de la teología contemporánea da testimonio por sí mismo de otras maneras de hablar de Dios, que no son filosóficas, al menos en el sentido occidental. Existen abundantes esfuerzos de tal fructificación: las teologías del llamado Tercer Mundo, teologías indias latinoamericana, asiáticas, africanas, las teologías de la mujer, las de los migrantes, las de aquellos que reflexionan su fe en contextos ecuménicos e interreligiosos.

Otros lenguajes se articulan desde las más variadas experiencias de sufrimiento de las innumerables víctimas que hoy ingresan en el escenario mundial para hacerse oír. Su expresión es como un clamor que brota desde abajo, desde el reverso de la historia, desde los márgenes. No sólo reclaman la restitución de derechos violentados. Muestran también que 
es posible hablar de Dios de otro modo, con otra lógica y con otros lenguajes.

\section{REFERENCIAS}

-Alberigo, G. (1985). Christianisme en tant qu'histoire. En M.-D. Chenu, Une école de Théologé: le Saulchoir (pp. 11-35). Paris: Cerf.

-Amaladoss, M. (2000). Vivir en libertad. Las teologias de la liberación del continente asiático. Estella (Navarra): Verbo Divino.

-Azcuy, V., \& García Bachmann, M. (2009). Mujeres haciendo teologías. Estudios de autoras en América Latina, el Caribe y Estados Unidos. Buenos Aires: Teologanda-San Pablo.

-Azcuy, V., García Bachamann, M., \& Lértora Mendoza, C. (2007). Mujeres haciendo teologías. Diccionario de obras de autoras en América Latina, el Caribe y Estados Unidos. Buenos Aires: Teologanda-San Pablo.

-Azcuy, V., Mazzini, M., \& Raimondo, N. (2008). Mujeres haciendo teologías. Antología de textos de autoras en América Latina, el Caribe y Estados Unidos. Buenos Aires: Teologanda-San Pablo.

-Bingemer, M. C. (1993). La pneumatologia como posibilidade de dialogo e missao universais. En F. L. Texeira, Dialogo dos Passaros (pp. 11-121). Sao Paulo: Paulinas.

-Bosch, J. (2004). Chenu, Marie-Dominique. En J. Bosch, Diccionario de teólogos/as con-temporáneos (pp. 251-265). Burgos: Monte Carmelo.

-Caram, M. J. (2008). Manifestación del Espíritu en la fe de los pueblos andinos. Ciencia Tomista, CXXXV(436), 319-360.

-Caram, M. J. (2012). Marie Dominique Chenu. Un teólogo en la historia. Anatéllei, XIV(28), 121-133.

-Caram, M. J. (2014). Marie Dominique Chenu, Une école de théologie: Le Saulchoir. Presentación de un ensayo teológico pionero. Páginas (233), 2030.

-Chenu, M.-D. (1985). Une école de théologie: le Saulchoir. Paris: Cerf.

-Chipana Quispe, S. (2012). Teología y Buen vivir. En Fundación Amerindia (Ed.), Congreso Continental de Teología. La Teología de la Liberación en prospectiva (Vol. II, págs. 231-262). Montevideo, Uruguay: Doble clic editoras.

-Concilio Vaticano II. (1965). Constitución Pastoral Gaudium et Spes. Sobre la Iglesia en el mundo actual. Recuperado de http://www.vatican.va/archive/hist_councils/ii_vatican_council/docume nts/vat-ii_const_19651207_gaudium-et-spes_sp.html

-De Prado, C. (1994). Yo siento a Dios de otro modo. Recuperdo de http://www.servicioskoinonia.org/agenda/archivo/obra.php?ncodigo $=10$ 0

-Dupuis, J. (2000). Hacia una teología cristiana del pluralismo religioso. Santander: Sal Terrae.

-Ferrater Mora, J. (1971). Diccionario de filosofía (Vol. III). Buenos Aires: Sudamericana. 
-Franco, A. (2007). Marie-Dominique Chenu. Madrid: San Pablo.

-Gutiérrez, G. (1995). Hablar de Dios desde el sufrimiento del inocente. Una reflexión sobre el libro de Job. Salamanca: Sígueme.

-Gutiérrez, G. (1996). Teología de la liberación. Perspectivas. Con una nueva introducción. Mirar lejos. Lima: CEP.

-Johnson, E. (2008). La búsqueda del Dios vivo. Trazar las fronteras de la teología de Dios. Santander: Sal Terrae.

-Ladrière, J. (1985). Théologie et historicité. En M.-D. Chenu, Une école de théologie: le Saulchoir (pp. 61-79). Paris: Cerf.

-López Hernández, E. (1993). Insurgencia teológica de los pueblos indios. Christus (7), 7-13.

-López Hernández, E. (2000). Teología India. Antología. Cochabamba; Buenos Aires: Universidad Católica Boliviana, Instituto Superior de Estudios Teológicos, Departamento de Misionología.

-López Hernández, E. (2014). La experiencia teologal indígena. Aporte a las iglesias. Recuperado de http://sedosmission.org/old/spa/hernandez.htm

-Pieris, A. (2001). Liberación, incuturación, diálogo interreligioso. Un nuevo paradigma desde Asia. Estella (Navarra): Verbo Divino.

-Roland-Gosselin, M.-D. (1932). Essai d'une étude critique de la connaissance. Introduction et première partie. Paris: Vrin.

-Scannone, J. C. (1990). Teología e interdisciplinariedad. Presencia del saber teológico en el ámbito de las Ciencias. Teológica Xaveriana (94), 63-79.

Sumario: Introducción; 1. Un derrotero inesperado; 2. La vida intelectual en Le Saulchoir, 3. Situación de la filosofía; 3.1. Filosofía tomista y escolástica barroca; 3.1.1. La adopción del concepto de philosophia perennis; 3.1.2. La concepción de lo inteligible; 3.1.3. La ontología; 3.2. Filosofía escolástica y filosofía moderna; 3.2.1. El humanismo; 3.2.2. La ciencia moderna; 3.2.3. La crítica kantiana; 3.2.4. La historia; 4. Teología y lenguaje filosófico; Conclusiones; Referencias. 